\title{
Discrimination of Members of the Family Pasteurellaceae Based on Polyamine Patterns
}

\author{
HANS-JÜRGEN BUSSE, ${ }^{1 *}$ SEBASTIAN BUNKA, ${ }^{2}$ ANDREAS HENSEL, ${ }^{1}$ \\ AND WERNER LUBITZ ${ }^{1}$ \\ Institut für Mikrobiologie und Genetik, Universität Wien, $A-1030$ Vienna, ${ }^{1}$ and \\ Institut für Medizinische Chemie, Veterinärmedizinische \\ Universität Wien, A-1210 Vienna, ${ }^{2}$ Austria
}

\begin{abstract}
In a study of the classification of members of the family Pasteurellaceae, the polyamine patterns of 101 strains were analyzed. These strains included the type strains of species belonging to the genera Actinobacillus, Haemophilus, and Pasteurella and additional strains of selected species, as well as numerous unnamed strains. Members of the genus Actinobacillus sensu stricto were characterized by the presence of 1,3-diaminopropane as the predominant compound. In the majority of the species of the genus Haemophilus sensu stricto 1,3diaminopropane was also the major compound in the polyamine pattern. In contrast, Haemophilus intermedius subsp. gazogenes and Haemophilus parainfluenzae were characterized by high levels of 1,3-diaminopropane, cadaverine, and putrescine. These results confirmed the findings of Dewhirst et al. (F. E. Dewhirst, B. J. Paster, I. Olsen, and G. J. Fraser, Zentralbl. Bakteriol. Parasitenkd. Infektionskr. Hyg. Abt. 1 Orig. 279:35-44, 1993), who demonstrated that $H$. parainfluenzae is phylogenetically only distantly related to the type species of the genus Haemophilus, Haemophilus influenzae. The phylogenetic diversity of the genus Pasteurella sensu stricto determined by Dewhirst et al. was also reflected to some extent by different polyamine patterns. The common characteristics found in Pasteurella multocida, Pasteurella canis, Pasteurella dagmatis, Pasteurella stomatis, and Pasteurella sp. strain B were high levels of putrescine and spermidine and the presence of the unusual triamine sym-norspermidine. Pasteurella avium, Pasteurella gallinarum, and Pasteurella volantium contained high concentrations of 1,3-diaminopropane and spermidine. Pasteurella langaa contained only high concentrations of 1,3-diaminopropane, and Pasteurella anatis was characterized by the presence of 1,3-diaminopropane as the predominant compound and high levels of putrescine and spermidine. Our data demonstrate that polyamine patterns are useful for discrimination within the family Pasteurellaceae.
\end{abstract}

The family Pasteurellaceae accomodates species classified in the genera Pasteurella Trevisan 1887 (31), Actinobacillus Brumpt 1910 (3), Haemophilus Winslow et al. 1917 (32), and Lonepinella Osawa et al. 1995 (24), as well as numerous unnamed strains. All members of the family are parasites of vertebrates, such as mammals, birds, and reptiles. Phylogenetically, the family is located in the gamma subclass of the Proteobacteria and clusters with facultatively anaerobic, gram-negative, chemoorganotrophic bacteria which are members of the families Enterobacteriaceae, Aeromonadaceae, and Vibrionaceae $(5,6,33)$.

Extensive DNA-DNA hybridization studies have demonstrated the genetic relatedness of members of the family Pasteurellaceae and have resulted in the definition of three genera, the genera Actinobacillus, Pasteurella, and Haemophilus (20). Recent DNA-rRNA hybridization studies and 16S rRNA sequence analyses of members of the family Pasteurellaceae have revealed that there is great heterogeneity within this family (6, 7). Many recognized species of the three genera are genetically more closely related to the type species of another genus than to the type species of their own genus. For example, Actinobacillus actinomycetemcomitans clusters phylogenetically with Haemophilus influenzae; Pasteurella bettyae, Pasteurella haemolytica, Haemophilus paraphrohaemolyticus, and Haemophilus ducreyi cluster phylogenetically with Actinobacillus lignieresii; and Haemophilus paragallinarum, Haemophilus haemoglobi-

* Corresponding author. Mailing address: Institut für Mikrobiologie und Genetik, Universität Wien, Dr. Bohr-Gasse 9, A-1030 Vienna, Austria. Phone: 43-1-79515-4121. Fax: 43-1-79515-4114. E-mail: elliot @gem.univie.ac.at. nophilus, and Haemophilus paracuniculus cluster phylogenetically with Pasteurella multocida. Sometimes no phylogenetic relationship between a species and any of the genera is found. In some cases, the results of DNA reassociation and 16S rRNA analyses somewhat controversarially indicate that an organism belongs to different groups. Actinobacillus capsulatus, Haemophilus parainfluenzae, Pasteurella langaa, and Pasteurella anatis are considered members of their respective genera sensu stricto based on DNA reassociation data (20), but each of these taxa is phylogenetically only distantly related to the type species of its genus (7). However, it is obvious from the results of these investigations that the family Pasteurellaceae encompasses more than the four genera that are recognized at this time.

Differentiation based on physiological and biochemical data often poses problems, as has been demonstrated for members of the genera Actinobacillus and Pasteurella, which are intermixed as determined by physiological and biochemical data $(11,30)$. Also, the present definition of the genus Haemophilus as a taxon whose members require $\mathrm{X}$ factor (protoporphyrin or protoheme) and/or V factor (NAD or NADP), combined with other phenotypic traits does not correlate with DNA reassociation data (16). Due to the lack of clear-cut definitions of the genera Actinobacillus, Pasteurella, and Haemophilus (including phenotypic and genotypic data), proposing new genera in the family is difficult. Thus, other approaches for classification of members of the family Pasteurellaceae are needed.

Attempts to define taxa in the family Pasteurellaceae based on analyses of fatty acids and polar lipids have failed due to the homogeneity of the fatty acids and polar lipids in representatives of the genera Actinobacillus, Haemophilus, and Pasteurella $(8,9,21)$. Whole-cell sugar analysis revealed heterogeneous 
patterns which allowed discrimination between groups or species $(8,9,21)$; the importance of this characteristic for genus discrimination, however, has not been demonstrated so far. Another important approach for differentiating taxa within the family is quinone content analysis, but there are problems in interpretation caused by contradictory results obtained for several species, such as $A$. actinomycetemcomitans, Haemophilus aphrophilus, and Pasteurella pneumotropica (9, 15, 21). Thus, well-established chemotaxonomic approaches do not appear to be suitable for reclassification of taxa within the family Pasteurellaceae.

Analysis of polyamine patterns has been shown to be a powerful method for classifying gram-negative bacteria $(1,4$, $12,13)$. In the recent proposal for establishment of the genus Lonepinella, promising results were obtained by using polyamine analysis for differentiation within the family Pasteurellaceae (24). However, the polyamine patterns of only the type species of the four genera were analyzed. Extension of the polyamine approach to more strains belonging to the family Pasteurellaceae will be necessary to evaluate the usefulness of polyamine patterns for classification within this family. In this study we examined polyamine data for the type strains of species belonging to the genera Haemophilus, Actinobacillus, and Pasteurella; the organisms used were selected mainly on the basis of their known positions in the family, as shown by DNA-DNA hybridization, DNA-rRNA hybridization, and/or $16 \mathrm{~S}$ rRNA sequence analyses. Also included were additional strains of several species, as well as many unnamed strains considered to be members of the family Pasteurellaceae.

\section{MATERIALS AND METHODS}

Strains used and culture conditions. A total number of 101 strains were included in this study (Tables 1 through 4 ). The majority of the strains were provided by W. Mannheim (Marburg, Germany). A. lignieresii 9/91, Actinobacillus suis A209/83, P. haemolytica A2683/81, and P. multocida $\mathrm{H} 983 / 87$ and H1161/87 were provided by G. Amtsberg (Hannover, Germany). Actinobacillus pleuropneumoniae ATCC $27088^{\mathrm{T}}$ and 56153 were donated by R. Nielsen (Copenhagen, Denmark). A. pleuropneumoniae $\mathrm{N}-273$ and N-282 were obtained from $\mathrm{E}$ Molnar (Budapest, Hungary). A. pleuropneumoniae PL2 and PL10 were provided by $P$. Kielstein (Jena, Germany), and $H$. paragallinarum $x \times 36$ and $x \times 37$ were provided by K.-H. Hinz (Hannover, Germany).

The cells were cultivated aerobically at $37^{\circ} \mathrm{C}$ on PPLO broth (Difco Laboratories, Detroit, Mich.) supplemented with $0.001 \%$ NAD (Sigma Chemical Co. St. Louis, Mo.), $0.001 \%$ hemin (Sigma), and 1\% IsoVitaleX Enrichment (Becton Dickinson Microbiology Systems, Cockeysville, Md.). H. ducreyi MCCM $00729^{\mathrm{T}}$ was grown at $30^{\circ} \mathrm{C}$. Cells were harvested in the logarithmic phase of growth when the optical density at $600 \mathrm{~nm}$ was approximately $70 \%$ of the maximum optical density at $600 \mathrm{~nm}$ determined for each strain.

Polyamine analysis. Polyamines were extracted as described previously (2) Approximately $40 \mathrm{mg}$ of lyophilized cells was hydrolyzed in $1 \mathrm{ml}$ of $0.2 \mathrm{~N}$ perchloric acid for $30 \mathrm{~min}$ at $100^{\circ} \mathrm{C}$ with occasional shaking. Each mixture contained the internal standard 1,8-diaminooctane $(25 \mu \mathrm{mol} / 40 \mathrm{mg}$ of cells) After extraction the samples were centrifuged, $0.2 \mathrm{ml}$ of each supernatant was transferred to a tube containing $0.3 \mathrm{ml}$ of an $\mathrm{Na}_{2} \mathrm{CO}_{3}$ solution $(100 \mathrm{mg} / \mathrm{ml})$, and $0.8 \mathrm{ml}$ of a dansyl chloride solution $(7.5 \mu \mathrm{g} / \mathrm{ml}$ in acetone) was added. The tube was closed tightly, and dansylation was performed for $20 \mathrm{~min}$ at $60^{\circ} \mathrm{C}$. Subsequently, $0.1 \mathrm{ml}$ of a proline solution $(50 \mathrm{mg} / \mathrm{ml})$ was added to bind the excess dansyl chloride during incubation for $10 \mathrm{~min}$ at $60^{\circ} \mathrm{C}$. After cooling to $5^{\circ} \mathrm{C}$, the polyamines were extracted with $0.1 \mathrm{ml}$ of toluene with shaking. The upper phase was used for a high-performance liquid chromatography analysis. The highperformance liquid chromatography apparatus was equipped with two Waters model 510 pumps, a model U6K injector, a column oven, a spectrofluorometric detector (Jasco model 821-FP), and a reversed-phase column ( 250 by $4 \mathrm{~mm}$; Hypersil octyldecyl silane; $5-\mu \mathrm{m}$ particles). To separate the polyamines, a linear 40 to $85 \%$ acetonitrile-water gradient was applied for $35 \mathrm{~min}$, followed by a linear 85 to $100 \%$ acetonitrile-water gradient applied for $15 \mathrm{~min}$ at $40^{\circ} \mathrm{C}$. To detect dansylated polyamines, an excitation wavelength of $360 \mathrm{~nm}$ was used, and the emitted light was measured at $520 \mathrm{~nm}$. Polyamine concentrations were calculated by using internal standards. Repeated analyses of selected samples demonstrated that the deviation for major compounds was less than $10 \%$.

\section{RESULTS AND DISCUSSION}

An extensive study of the phylogeny of members of the Pasteurellaceae based on $16 \mathrm{~S}$ rRNA sequence analyses demonstrated that the family can be divided into seven clusters and several subclusters (7). The type species of the genera Actinobacillus, Haemophilus, Pasteurella, and Lonepinella are each in a separate group, which demonstrates the phylogenetic distinctness of these taxa $(7,24)$. Below, the results of the polyamine analyses (Tables 1 through 4 ) are discussed according to the phylogenetic clustering of the strains (7), and strains whose phylogenetic positions are not known are discussed separately (Table 4).

Cluster 1. Cluster 1 is subdivided into three subclusters. The only representative of subcluster $1 \mathrm{~A}$ is $A$. actinomycetemcomitans, which includes four strains (7). This species has been reclassified several times $(27,28)$. The only significant compound in the polyamine pattern of the type strain, $A$. actinomycetemcomitans MCCM 00145, was 1,3-diaminopropane. This pattern was also found in species belonging to subcluster 1B (H. aphrophilus MCCM $00203^{\mathrm{T}}$ and Haemophilus segnis MCCM 01957 ${ }^{\mathrm{T}}$ ) and subcluster 1C (H. influenzae MCCM $00591^{\mathrm{T}}$ and Haemophilus aegyptius MCCM 02771 ${ }^{\mathrm{T}}$ ). DNA reassociation studies (20) have demonstrated that the type strain of the type species of the genus Haemophilus, $H$. influenzae (MCCM 00591), and the type strain of $H$. aegyptius (MCCM 02772) are closely related and are considered members of the genus Haemophilus sensu stricto, whereas $H$. aphrophilus MCCM $00203^{\mathrm{T}}$ and $H$. segnis MCCM $01957^{\mathrm{T}}$ are not included in the genus Haemophilus sensu stricto (20). These data may provide evidence that members of the genus Haemophilus are characterized by the presence of 1,3-diaminopropane as the predominant compound, while a different polyamine pattern indicates that an organism does not belong to this genus. This homogeneity in the polyamine patterns of the species belonging to cluster 1 , in addition to the similar quinone compositions of these organisms (9), indicates that there is a close phylogenetic relationship and may support inclusion of $A$. actinomycetemcomitans, $H$. aphrophilus, and $H$. segnis in the genus Haemophilus sensu stricto.

Cluster 2. The polyamine patterns of the cluster 2, subcluster $2 \mathrm{~A}$ organisms Actinobacillus seminis MCCM $00565^{\mathrm{T}}$, Pasteurella mairii MCCM $00633^{\mathrm{T}}$, and Pasteurella aerogenes MCCM $00402^{\mathrm{T}}$ were analyzed (7). The polyamine patterns of the first two organisms resembled each other, whereas $P$. aerogenes MCCM $00402^{\mathrm{T}}$ had a different polyamine pattern (Tables 1 and 3). The differences may be explained by the deep phylogenetic branching of $P$. aerogenes within the subcluster (7). Thus, the grouping of $P$. aerogenes with $P$. mairii MCCM $00633^{\mathrm{T}}$ and $A$. seminis MCCM $00565^{\mathrm{T}}$ in a genuslike structure needs to be confirmed by other data. These observations confirm that $P$. aerogenes MCCM $00402^{\mathrm{T}}$ (19) should be excluded from the genus Pasteurella sensu stricto (see below) and also are not consistent with classification of $P$. mairii $\mathrm{MCCM}$ $00633^{\mathrm{T}}$ in the genus Pasteurella sensu stricto and classification of $A$. seminis MCCM $00565^{\mathrm{T}}$ in the genus Actinobacillus. The polyamine compositions of two strains that were obtained as $P$. aerogenes-like strain MCCM 01523 and $P$. aerogenes complex strain MCCM 01544 differed significantly from the polyamine compositions of $P$. aerogenes MCCM $00402^{\mathrm{T}}$ and the species of the genus Pasteurella sensu stricto (Table 3); this finding demonstrates that these two strains cannot be considered members of $P$. aerogenes or the genus Pasteurella sensu stricto.

Cluster 3. Species belonging to the genus Pasteurella sensu stricto (19) fall into three of the four subclusters of cluster 3. The type species of the genus, $P$. multocida, is located in sub- 


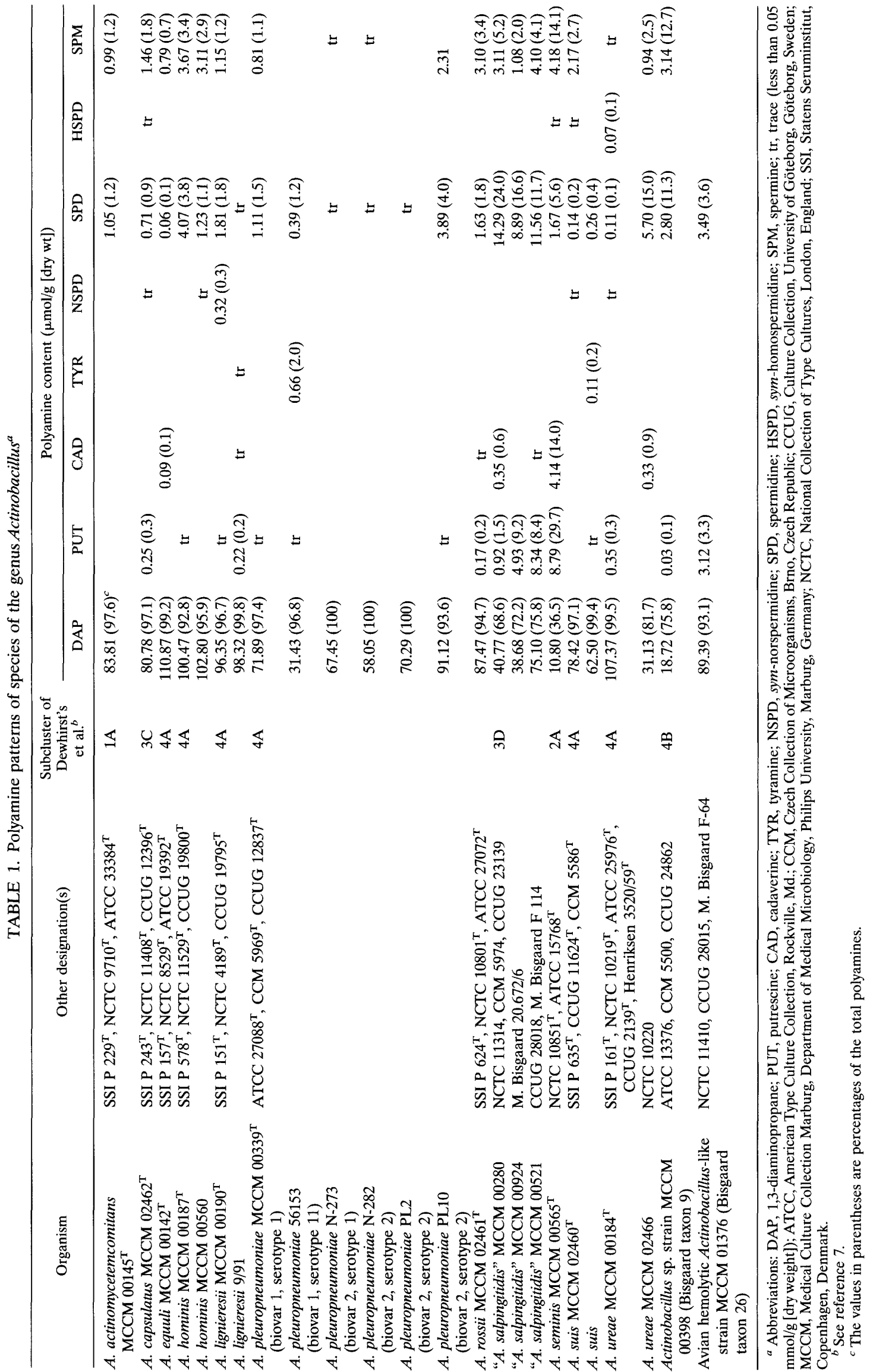


TABLE 2. Variation in the polyamine patterns of selected members of the genus Haemophilus ${ }^{a}$

\begin{tabular}{|c|c|c|c|c|c|c|c|c|c|c|}
\hline \multirow{2}{*}{ Organism } & \multirow{2}{*}{ Other designation(s) } & \multirow{2}{*}{$\begin{array}{l}\text { Cluster or subcluster } \\
\text { of Dewhirst's et al. }\end{array}$} & \multicolumn{8}{|c|}{ Polyamine content $(\mu \mathrm{mol} / \mathrm{g}$ [dry wt]) } \\
\hline & & & DAP & PUT & CAD & TYR & NSPD & SPD & HSPD & SPM \\
\hline H. aegyptius MCCM $02772^{\mathrm{T}}$ & NCTC $8502^{\mathrm{T}}$, ATCC $11116^{\mathrm{T}}$ & $1 \mathrm{C}$ & $35.56(91.4)^{c}$ & $3.16(8.2)$ & $0.19(0.5)$ & & & $\operatorname{tr}$ & & $\operatorname{tr}$ \\
\hline H. aegyptius MCCM 00678 & NCTC 8134 & & $28.51(62.9)$ & $10.11(22.3)$ & $6.67(14.7)$ & & & & & \\
\hline H. aphrophilus MCCM $00203^{\mathrm{T}}$ & $\begin{array}{l}\text { SSI P } 801^{\mathrm{T}} \text {, NCTC } 5906^{\mathrm{T}} \text {, } \\
\text { ATCC } 33389^{\mathrm{T}}\end{array}$ & 1B & $24.10(98.2)$ & $0.11(0.4)$ & $0.05(0.2)$ & & & $0.22(0.9)$ & & $0.06(0.2)$ \\
\hline H. ducreyi MCCM $00729^{\mathrm{T}}$ & $\begin{array}{l}\text { NCTC } 10945^{\mathrm{T}}, \text { ATCC } 33940^{\mathrm{T}}, \\
\text { CCUG } 4438^{\mathrm{T}}, \text { CIP } 54.2^{\mathrm{T}}\end{array}$ & $4 \mathrm{~B}$ & $59.51(79.1)$ & $0.06(0.1)$ & $0.38(0.5)$ & & $\operatorname{tr}$ & $8.65(11.5)$ & & $6.68(8.9)$ \\
\hline “H. felis” MCCM 02738 & ATCC $49733^{\mathrm{T}}$ & & $0.43(2.5)$ & $12.03(70.4)$ & $0.95(5.6)$ & & $3.03(17.7)$ & $0.64(3.7)$ & & $\operatorname{tr}$ \\
\hline “H. felis” МССМ 02064 & CCUG 30904 & & $7.21(33.4)$ & $0.42(1.9)$ & & & $8.62(40.0)$ & $4.42(20.5)$ & & $0.90(4.2)$ \\
\hline $\begin{array}{l}\text { H. influenzae MCCM } 00591^{\mathrm{T}} \\
\text { (biovar II) }\end{array}$ & $\begin{array}{l}\text { NCTC } 8143^{\mathrm{T}}, \text { ATCC } 33391^{\mathrm{T}}, \\
\text { CCUG } 15643^{\mathrm{T}}\end{array}$ & $1 \mathrm{C}$ & $55.08(96.2)$ & $0.76(1.3)$ & & $0.54(0.9)$ & & $0.79(1.4)$ & & $0.08(0.1)$ \\
\hline $\begin{array}{l}\text { H. intermedius subsp. gazogenes } \\
\text { MCCM } 02082\end{array}$ & CCUG 15793 & & $5.13(29.1)$ & $7.46(42.4)$ & $4.97(28.2)$ & & & $0.05(0.3)$ & & \\
\hline H. paracuniculus $\mathrm{MCCM} 00703^{\mathrm{T}}$ & ATCC $29986^{\mathrm{T}}$, CCUG $15644^{\mathrm{T}}$ & $3 \mathrm{C}$ & $50.96(71.6)$ & $0.17(0.2)$ & $0.14(0.2)$ & & $\operatorname{tr}$ & $6.61(9.3)$ & $0.6(0.8)$ & $12.71(17.9)$ \\
\hline H. paragallinanum $\mathrm{MCCM} 00300^{\mathrm{T}}$ & $\begin{array}{l}\text { NCTC } 11296^{\mathrm{T}}, \text { ATCC } 29545^{\mathrm{T}}, \\
\text { CCUG } 12835^{\mathrm{T}}\end{array}$ & $3 \mathrm{~A}$ & $48.72(84.9)$ & $0.17(0.3)$ & $0.37(0.6)$ & & $\operatorname{tr}$ & $5.21(9.1)$ & tr & $2.91(5.1)$ \\
\hline H. paragallinarum $\times x 36$ & & & $45.54(73.7)$ & $1.58(2.6)$ & $0.31(0.5)$ & & & $12.66(20.5)$ & & $1.66(2.7)$ \\
\hline H. paragallinarum $\times x 37$ & & & $52.67(70.6)$ & $2.44(3.3)$ & $\operatorname{tr}$ & & & $18.40(24.7)$ & & $1.09(1.5)$ \\
\hline H. parahaemolyticus MCCM $00504^{\mathrm{T}}$ & $\begin{array}{l}\text { NCTC } 8479^{\mathrm{T}}, \text { ATCC } 10014^{\mathrm{T}}, \\
\text { CCUG } 3716^{\mathrm{T}}\end{array}$ & $4 \mathrm{~A}$ & $18.79(86.7)$ & & & & & $1.19(5.5)$ & & $1.68(7.8)$ \\
\hline H. parainfluenzae $\mathrm{MCCM} 00486^{\mathrm{T}}$ & $\begin{array}{l}\text { NCTC } 7857^{\mathrm{T}}, \text { ATCC } 33392^{\mathrm{T}}, \\
\text { CCUG } 12836^{\mathrm{T}}\end{array}$ & 6 & $14.85(40.2)$ & $7.87(21.3)$ & $14.21(39.5)$ & & & & & \\
\hline H. parasuis MCCM $02736^{\mathrm{T}}$ & $\begin{array}{l}\text { NCTC } 4557^{\mathrm{T}}, \text { ATCC } 19417^{\mathrm{T}}, \\
\text { CCUG } 3712^{\mathrm{T}}\end{array}$ & $3 \mathrm{C}$ & $41.01(65.4)$ & $20.33(32.4)$ & $0.27(0.4)$ & & & $1.06(1.7)$ & & \\
\hline H. segnis $\mathrm{MCCM} 01957^{\mathrm{T}}$ & $\begin{array}{l}\text { NCTC } 10977^{\mathrm{T}}, \text { ATCC } 33393^{\mathrm{T}}, \\
\quad \text { CCUG } 10787^{\mathrm{T}}\end{array}$ & 1B & $34.52(99.7)$ & & & & & $0.11(0.3)$ & & \\
\hline $\begin{array}{l}\text { Avian Haemophilus-like strain } \\
\text { MCCM } 01034\end{array}$ & & & $25.78(62.2)$ & $0.27(0.7)$ & $0.19(0.5)$ & & & $10.66(25.7)$ & & $4.58(11.0)$ \\
\hline $\begin{array}{l}\text { Avian Haemophilus-like strain } \\
\text { MCCM } 02795\end{array}$ & & & $0.14(0.5)$ & $3.96(14.2)$ & & & & $21.68(77.8)$ & & $2.10(7.5)$ \\
\hline
\end{tabular}

a CIP, Collection de l'Institut Pasteur, Paris, France. For other abbreviations see Table 1, footnote $a$.

See reference 7 .

${ }^{c}$ The values in parentheses are percentages of the total polyamines. 
TABLE 3. Variation in the polyamine patterns of selected members of the genus Pasteurella ${ }^{a}$

\begin{tabular}{|c|c|c|c|c|c|c|c|c|c|c|}
\hline \multirow{2}{*}{ Organism } & \multirow{2}{*}{ Other designation(s) } & \multirow{2}{*}{$\begin{array}{c}\text { Cluster or } \\
\text { subcluster of } \\
\text { Dewhirst et al. }{ }^{b}\end{array}$} & \multicolumn{8}{|c|}{ Polyamine content $(\mu \mathrm{mol} / \mathrm{g}[\mathrm{dry} \mathrm{wt}])$} \\
\hline & & & DAP & PUT & CAD & TYR & NSPD & SPD & HSPD & SPM \\
\hline P. aerogenes MCCM $00402^{\mathrm{T}}$ & SSI P $634^{\mathrm{T}}$, ATCC $27883^{\mathrm{T}}$, CCUG $9995^{\mathrm{T}}$ & $2 \mathrm{~A}$ & $0.49(1.6)^{c}$ & $6.52(21.2)$ & $1.76(5.7)$ & $0.51(1.7)$ & & $19.74(64.2)$ & & $1.72(5.6)$ \\
\hline P. aerogenes-like strain MCCM 01523 & & & $38.27(88.6)$ & $3.44(8.0)$ & $1.33(3.1)$ & & $\operatorname{tr}$ & $0.13(0.3)$ & & $\operatorname{tr}$ \\
\hline P. aerogenes complex strain MCCM 01544 & & & $40.34(91.0)$ & $0.12(0.3)$ & $0.23(0.5)$ & & & $2.49(5.6)$ & & $1.15(2.6)$ \\
\hline P. anatis MCCM $00140^{\mathrm{T}}$ & 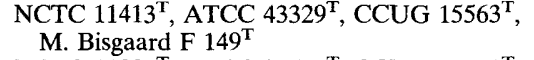 & 3D & $50.84(56.0)$ & $22.48(24.8)$ & $0.09(0.1)$ & & & $14.05(15.5)$ & & $3.26(3.6)$ \\
\hline P. avium MCCM $00272^{\mathrm{T}}$ & NCTC $11297^{\mathrm{T}}$, ATCC $29546^{\mathrm{T}}$, CCUG $12833^{\mathrm{T}}$ & $3 \mathrm{~A}$ & $45.38(74.0)$ & $1.74(2.8)$ & & & & $12.08(19.7)$ & & $2.11(3.4)$ \\
\hline P. bettyae $\mathrm{MCCM} 00450^{\mathrm{T}}$ & $\begin{array}{l}\text { SSI P } 843^{\mathrm{T}}, \text { NCTC } 10535^{\mathrm{T}}, \text { ATCC } 23273^{\mathrm{T}} \text {, } \\
\text { CCUG } 2042^{\mathrm{T}}\end{array}$ & 4B & $48.21(94.0)$ & $0.52(1.0)$ & $0.14(0.3)$ & & & $2.14(4.2)$ & & $0.29(0.6)$ \\
\hline P. caballi $\mathrm{MCCM} 00841^{\mathrm{T}}$ & ATCC $49197^{\mathrm{T}}$ & & $67.72(88.7)$ & $5.98(7.8)$ & $2.59(3.4)$ & & & $0.07(0.1)$ & & \\
\hline P. canis MCCM $00045^{\mathrm{T}}$ & $\begin{array}{l}\text { SSI P } 293^{\mathrm{T}}, \text { NCTC }^{2} 11621^{\mathrm{T}}, \text { ATCC } 43326^{\mathrm{T}} \text {, } \\
\quad \text { CCUG } 12400^{\mathrm{T}}\end{array}$ & 3B & $0.36(1.0)$ & $12.78(34.8)$ & $2.72(7.4)$ & & $0.77(2.1)$ & $18.87(51.3)$ & & $1.27(3.5)$ \\
\hline P. canis biotype 1 strain MCCM 00047 & & & $7.40(12.2)$ & $18.16(29.9)$ & $3.93(6.5)$ & & $1.77(2.9)$ & $26.69(44.0)$ & & $2.71(4.5)$ \\
\hline P. canis MCCM 00927 & & & $0.41(1.5)$ & $17.09(63.0)$ & $3.59(13.2)$ & & $1.36(5.0)$ & $3.92(14.4)$ & & $0.76(2.8)$ \\
\hline P. dagmatis MCCM $00024^{\mathrm{T}}$ & 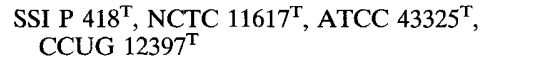 & $3 \mathrm{~B}$ & $19.65(33.5)$ & $26.95(46.0)$ & $0.27(0.5)$ & & $3.45(5.9)$ & $8.19(14.0)$ & & $0.07(0.1)$ \\
\hline P. dagmatis MCCM 00558 & & & $5.15(6.6)$ & $51.12(65.0)$ & tr & & $4.52(5.7)$ & $17.08(21.7)$ & & $0.74(0.9)$ \\
\hline P. gallinarum MCCM $00031^{\mathrm{T}}$ & 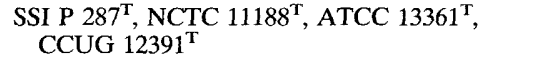 & $3 \mathrm{~A}$ & $37.48(76.2)$ & $0.08(0.2)$ & $0.12(0.2)$ & & & $7.18(14.6)$ & & $4.32(8.8)$ \\
\hline$P$. gallinarum MCCM 000 & & & $4.69(7.4)$ & $18.73(2$ & $0.61(1.0)$ & & $5.48(8.6)$ & 31.40 & & $2.75(4.3)$ \\
\hline P. granulomatis MCCM $00576^{\mathrm{T}}$ & SSI P $1135^{\mathrm{T}}$ & & $69.86(95.2)$ & $0.59(0.8)$ & $0.12(0.2)$ & & & $1.26(1.7)$ & & $1.58(2.2)$ \\
\hline P. haemolytica MCCM $00395^{\mathrm{T}}$ & $\begin{array}{l}\text { SSI P } 586^{\mathrm{T}} \text {, NCTC } 9380^{\mathrm{T}}, \text { ATCC } 33396^{\mathrm{T}} \text {, } \\
\text { CCUG } 408^{\mathrm{T}}\end{array}$ & 4B & $67.87(93.9)$ & $0.76(1.1)$ & & & & $1.86(2.6)$ & & $1.79(2.5)$ \\
\hline P. haemolytica MCCM 00388 (third taxon) & SSI P 731 & & $61.74(77.6)$ & $1.48(1.9)$ & $0.25(0.3)$ & & & $9.20(11.6)$ & & $6.87(8.6)$ \\
\hline P. haemolytica MCCM 00594 (fourth taxon) & SSI P 955 & & $56.90(78.9)$ & $1.09(1.5)$ & $0.29(0.4)$ & & & $4.61(6.4)$ & & $9.26(12.8)$ \\
\hline P. haemolytica A2683 & & & $90.34(97.2)$ & & & & & $1.16(1.2)$ & & $1.45(1.6)$ \\
\hline P. langaa $\mathrm{MCCM} 00120^{\mathrm{T}}$ & NCTC $11411^{\mathrm{T}}$, ATCC $43328^{\mathrm{T}}$, CCUG $15566^{\mathrm{T}}$ & 3D & $53.08(95.5)$ & $1.49(2.7)$ & & & & $1.04(1.9)$ & $\operatorname{tr}$ & $\operatorname{tr}$ \\
\hline "P. leonis" МСCM 00659 & SSI P 876 & & $15.24(24.6)$ & $29.27(47.3)$ & $0.60(1.0)$ & & $11.08(17.9)$ & $5.20(8.4)$ & & $0.53(0.9)$ \\
\hline P. mairii MCCM $00633^{\mathrm{T}}$ & SSI P $637^{\mathrm{T}}$, NCTC $10699^{\mathrm{T}}$, CCUG $27189^{\mathrm{T}}$ & $2 \mathrm{~A}$ & $49.81(47.7)$ & $44.89(43.0)$ & $9.48(9.10)$ & & & $0.10(0.1)$ & & $0.12(0.1)$ \\
\hline P. multocida subsp. multocida $\mathrm{MCCM} 00005^{\mathrm{T} d}$ & $\begin{array}{l}\text { SSI P } 427^{\mathrm{T}}, \text { NCTC } 10322^{\mathrm{T}}, \text { ATCC } 43137^{\mathrm{T}} \text {, } \\
\quad \text { CCUG } 17976^{\mathrm{T}}\end{array}$ & 3B & & $52.71(72.5)$ & $6.68(9.2)$ & & $1.38(1.9)$ & $11.57(15.9)$ & & $0.35(0.5)$ \\
\hline P. multocida subsp. gallicida $\mathrm{MCCM} 00021^{\mathrm{Td}}$ & SSI P $427^{\mathrm{T}}$, NCTC $10204^{\mathrm{T}}$, CCUG $17979^{\mathrm{T}}$ & & $0.97(1.2)$ & $56.23(67.0)$ & $2.25(2.7)$ & & $2.84(3.4)$ & $19.30(23.0)$ & & $2.39(2.8)$ \\
\hline P. multocida subsp. septica $\mathrm{MCCM} 00008^{\mathrm{T} d}$ & CCUG $19977^{\mathrm{T}}$, CIP A $125^{\mathrm{T}}$ & & $1.19(5.2)$ & $10.82(46.9)$ & $0.31(1.3)$ & & $1.71(7.4)$ & $7.20(31.2)$ & & $1.82(7.9)$ \\
\hline P. multocida H $1161 / 87$ & & & & $23.30(81.7)$ & $0.11(0.4)$ & & $0.26(0.9)$ & $4.31(15.1)$ & & $0.55(1.9)$ \\
\hline P. multocida H 983/87 & & & $4.87(7.4)$ & $8.76(13.3)$ & $1.32(2.0)$ & & $5.82(8.8)$ & $41.82(63.5)$ & & $3.27(5.0)$ \\
\hline $\begin{array}{l}\text { P. pneumotropica type Jawetz strain } \\
\text { MCCM } 00234^{\mathrm{T}}\end{array}$ & $\begin{array}{l}\text { SSI P } 421^{\mathrm{T}} \text {, NCTC } 8141^{\mathrm{T}} \text {, ATCC } 35149^{\mathrm{T}} \text {, } \\
{\text { CCUG } 12398^{\mathrm{T}}}^{\text {N }}\end{array}$ & 5 & $45.04(64.3)$ & $22.71(32.5)$ & $2.09(3.0)$ & & & $0.12(0.2)$ & & $0.10(0.1)$ \\
\hline P. pneumotropica complex strain MCCM 01911 & M. Kilian HK 366 & & $38.16(87.2)$ & $4.24(9.7)$ & $0.92(2.1)$ & & & $0.06(0.1)$ & & $0.39(0.9)$ \\
\hline P. pneumotropica type Heyl strain MCCM 00237 & SSI P 309, ATCC 12555, CCUG 998 & & $51.07(55.6)$ & $9.20(10.0)$ & $31.52(34.3)$ & & & & & $\operatorname{tr}$ \\
\hline P. pneumotropica type Heyl strain MCCM 00253 & & & $33.13(72.9)$ & $1.67(3.7)$ & $10.25(22.6)$ & & & $0.10(0.2)$ & & $0.30(0.7)$ \\
\hline Pasteurella species A strain MCCM 00072 & & & $32.60(68.5)$ & $0.17(0.4)$ & $0.05(0.1)$ & & & $11.09(23.3)$ & & $3.66(7.7)$ \\
\hline Pasteurella species B st & SSI P 683, CCUG 19794 & 3B & $3.02(5.5)$ & $10.64(19.3)$ & $1.32(2.4)$ & & $2.20(4.0)$ & $33.31(60.5)$ & & $4.61(8.4)$ \\
\hline P. stomatis $\mathrm{MCCM} 00100^{\mathrm{T}}$ & NCTC $11623^{\mathrm{T}}$, ATCC $43327^{\mathrm{T}}$, CCUG $17979^{\mathrm{T}}$ & 3B & $2.59(4.4)$ & $27.11(45.7)$ & $0.26(0.4)$ & $\operatorname{tr}$ & $3.55(6.0)$ & $23.34(39.4)$ & & $2.43(4.1)$ \\
\hline P. stomatis MCCM 00104 & NCTC 11624 & & tr & $28.40(58.1)$ & $0.10(0.2)$ & & $1.85(3.8)$ & $16.82(34.4)$ & & $1.70(3.5)$ \\
\hline P. stomatis MCCM 00490 & SSI P 294 & & $1.74(4.6)$ & $18.93(49.9)$ & $0.10(0.3)$ & $\operatorname{tr}$ & $0.96(2.5)$ & $14.44(38.1)$ & & $1.78(4.7)$ \\
\hline P. testudinis MCCM $00484^{\mathrm{T}}$ & ATCC $33688^{\mathrm{T}}$, CCUG $19802^{\mathrm{T}}$ & 7 & $0.47(0.7)$ & $16.80(26.5)$ & $0.27(0.4)$ & & & $40.83(64.5)$ & & $4.95(7.8)$ \\
\hline P. testudinis MCCM 00400 & & & $0.47(0.9)$ & $6.64(12.6)$ & $0.11(0.2)$ & & & $42.20(80.4)$ & & $3.10(5.9)$ \\
\hline P. trehalosi MCCM $02084^{\mathrm{T}}$ & NCTC $10370^{\mathrm{T}}$, ATCC $29703^{\mathrm{T}}$ & $3 \mathrm{C}$ & $62.86(96.7)$ & & & & & $1.81(2.8)$ & & $0.33(0.5)$ \\
\hline P. volantium MCCM $02204^{\mathrm{T}}$ & NCTC $3438^{\mathrm{T}}$, ATCC $14385^{\mathrm{T}}$, CCUG $3713^{\mathrm{T}}$ & $3 \mathrm{~A}$ & $29.95(74.8)$ & & $\operatorname{tr}$ & & & $6.79(17.0)$ & & $3.30(8.2)$ \\
\hline Pasteurella-like strain MCCM 00658 & NCTC 11051 & & $31.00(93.0)$ & & & & & $1.26(3.8)$ & & $1.06(3.2)$ \\
\hline Pasteurella-like & up 1 I. Kun & & $49.45(92.9)$ & $3.10(5.8)$ & $0.11(0.2)$ & & & $0.42(0.8)$ & $\operatorname{tr}$ & $0.17(0.3)$ \\
\hline Pasteurella-like strain MCCM 02120 & Hamster group 2 I. Kunstyr 507 & & $42.66(94.3)$ & $0.85(1.9)$ & & & & $0.14(0.3)$ & & $1.59(3.5)$ \\
\hline Pasteurella-like strain MCCM 01664 & J. Perrin D-578 & & $19.75(24.9)$ & $48.65(61.5)$ & $2.91(3.7)$ & & & $4.21(5.3)$ & & $3.64(4.6)$ \\
\hline
\end{tabular}

${ }^{a}$ For abbreviations see Table 1, footnote $a$, and Table 2, footnote $a$.

${ }^{b}$ See reference 7 .

$c$ The values in parentheses are percentages of the total polyamines.

${ }^{d}$ In addition, a small peak was detected in front of spermidine; this peak was assumed to be sym-norspermine. 


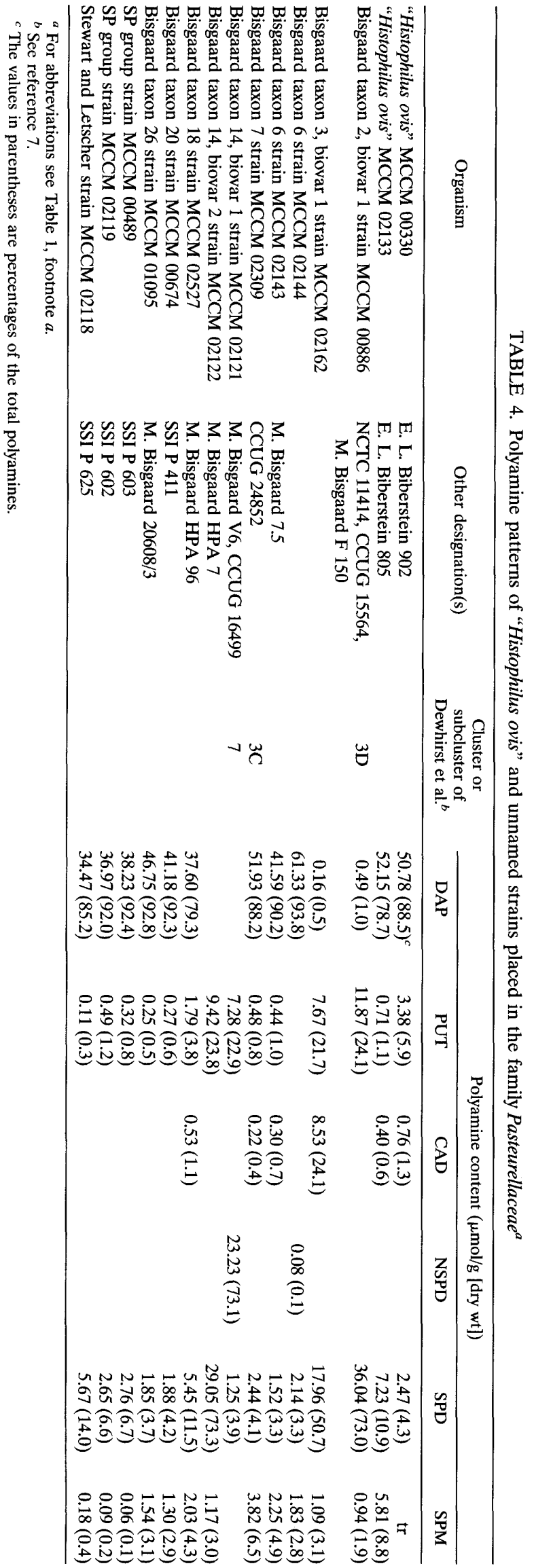

cluster 3B. A characteristic polyamine pattern containing the unusual triamine sym-norspermidine was obtained for $P$. multocida subsp. multocida MCCM $00005^{\mathrm{T}}, P$. multocida subsp. gallicida MCCM 00021 ${ }^{\mathrm{T}}$, P. multocida subsp. septica MCCM $00005^{\mathrm{T}}$, Pasteurella canis MCCM 00045 ${ }^{\mathrm{T}}$, Pasteurella dagmatis MCCM 00024 ${ }^{\mathrm{T}}$, Pasteurella stomatis MCCM 00100 ${ }^{\mathrm{T}}$, and Pasteurella species B strain MCCM $00102^{\mathrm{T}}$ (Table 3), which are grouped together in subcluster 3B. The polyamine pattern of $P$. dagmatis MCCM $00024^{\mathrm{T}}$ was slightly different. The characteristic features of this group were also detected in $P$. multocida $\mathrm{H}$ 1161/87 and H 983/87, P. canis MCCM 00047, and MCCM 00927, $P$. dagmatis MCCM 00558, and P. stomatis MCCM 00104 and MCCM 00490. No other strain analyzed in this study and placed in another genetic group in the family Pasteurellaceae contained the triamine sym-norspermidine in significant amounts. Thus, the presence of sym-norspermidine might be a diagnostic feature of subcluster $3 \mathrm{~B}$.

Other species considered to be members of the genus Pasteurella sensu stricto, including Pasteurella gallinarum, Pasteurella volantium, Pasteurella avium, $P$. langaa, $P$. anatis, and Pasteurella species A (19), could be easily distinguished from the $P$. multocida group (subcluster $3 \mathrm{~B}$ ) based on a lack of sym-norspermidine. These species have been shown to belong to subcluster $3 \mathrm{~A}$ (which includes $P$. gallinarum $\mathrm{MCCM} 00031^{\mathrm{T}}$, $P$. volantium MCCM $02204^{\mathrm{T}}, P$. avium MCCM $00272^{\mathrm{T}}, P a s-$ teurella species A strain MCCM 00072) and subcluster 3D (which includes $P$. langaa MCCM $00120^{\mathrm{T}}$ and $P$. anatis MCCM $\left.00140^{\mathrm{T}}\right)(7)$.

The Pasteurella species belonging to subcluster $3 \mathrm{~A}$ contained the predominant compound 1,3-diaminopropane (68 to $76 \%$ of the total polyamines) and high levels of spermidine (14 to $23 \%$ ) (Table 3 ). $H$. paragallinarum $\mathrm{MCCM} 00300^{\mathrm{T}}$, another member of subcluster $3 \mathrm{~A}$, had a similar pattern (Table 2). The relative levels of the single polyamines differed slightly in the type strain of $H$. paragallinarum, whereas the polyamine contents of two other $H$. paragallinarum isolates, $\mathrm{xx} 36$ and $\mathrm{xx} 37$, were within the ranges found for the Pasteurella species that grouped in cluster 3A. $P$. gallinarum MCCM 00033 had a polyamine pattern that was characteristic for members of subcluster 3B but different from the pattern found for members of subcluster $3 \mathrm{~A}$. The results of a biochemical and physiological characterization of $P$. gallinarum MCCM 00033 and a comparison with $P$. gallinarum MCCM $00031^{\mathrm{T}}$ did not indicate relatedness at the species level (data not shown). Thus, the identity of $P$. gallinarum MCCM 00033 should be checked, and the relationship of this organism to members of subcluster $3 \mathrm{~B}$ should be investigated.

Subcluster 3D consists of $P$. anatis, $P$. langaa, "Actinobacillus salpingitidis," Bisgaard taxon 2, and Bisgaard taxon 3. P. anatis MCCM $00140^{\mathrm{T}}$ and $P$. langaa $\mathrm{MCCM} 00120^{\mathrm{T}}$ are phylogenetically related only at a low level and differ in quinone composition (8). The quinone composition of $P$. anatis MCCM $00140^{\mathrm{T}}$ is very similar to the quinone composition of species belonging to subclusters $3 \mathrm{~A}$ and $3 \mathrm{~B}$. In this respect, $P$. langaa MCCM $00120^{\mathrm{T}}$ is not similar to other members of the genus Pasteurella sensu stricto. A close phylogenetic relationship between $P$. anatis MCCM $00140^{\mathrm{T}}$ and subcluster $3 \mathrm{~A}$ has been demonstrated recently, whereas $P$. langaa is located on a separate branch (10). The polyamine pattern of $P$. anatis MCCM $00140^{\mathbf{T}}$ (Table 3) distinguished this species from members of subcluster $3 \mathrm{~A}$ only because a high concentration of putrescine was found. $P$. langaa MCCM $00120^{\mathrm{T}}$ could be distinguished from the other species of the genus Pasteurella sensu stricto based on the presence of the predominant compound 1,3diaminopropane. Thus, the relationship of $P$. langaa to the genus Pasteurella sensu stricto needs to be reinvestigated. " $A$. 
salpingitidis" MCCM 00280 is not considered a member of the genus Actinobacillus sensu stricto (20). Phylogenetically, this organism is the organism that is most closely related to $P$. anatis. The suggested type strain of "A. salpingitidis" MCCM 00280 (25), had a polyamine pattern similar to the polyamine patterns found for strains belonging to subcluster $3 \mathrm{~A}$. In two strains obtained as " $A$. salpingitidis", MCCM 00924 and MCCM 00521, the putrescine content was somewhat higher than the putrescine content of the suggested type strain. The latter strain has been shown not to belong to the species " $A$. salpingitidis" (25). However, the polyamine patterns clearly differentiate the three strains from the genus Actinobacillus sensu stricto (see below) and indicate that the strains might be classified with the subcluster $3 \mathrm{~A}$ strains. Bisgaard taxon 2 strain MCCM 00886 and Bisgaard taxon 3 strain MCCM 02162 were characterized by polyamine patterns which distinguished them from the other members of subcluster 3D (Table 4). The heterogeneity of the polyamine patterns of the subcluster 3D strains indicates that this subcluster does not represent a group of strains that might be classified in one genus.

$H$. paracuniculus MCCM $00703^{\mathrm{T}}$, which grouped in subcluster $3 \mathrm{C}$, was characterized by a polyamine pattern (Table 2) which was similar to the polyamine pattern found in members of subcluster 3A. Other strains placed in subcluster 3C, including $A$. capsulatus MCCM $02462^{\mathrm{T}}$, Haemophilus parasuis MCCM $02736^{\mathrm{T}}$, Pasteurella trehalosi MCCM 02084 $4^{\mathrm{T}}$, and Bisgaard taxon 7 strain MCCM 02309, could be clearly distinguished from strain MCCM $00703^{\mathrm{T}}$. The polyamine pattern of $P$. trehalosi MCCM $2084^{\mathrm{T}}$ distinguished this organism from members of the genus Pasteurella sensu stricto (Table 3). A similar pattern was obtained for Bisgaard taxon 7 strain MCCM 02309 (Table 4) and $A$. capsulatus MCCM $02462^{\mathrm{T}}$ (Table 1 ). The predominant compound in the polyamine patterns, 1,3-diaminopropane, is common to the vast majority of the members of the family Pasteurellaceae and does not indicate relatedness to a certain cluster. On the other hand, based on the polyamine pattern, it cannot be assumed that Bisgaard taxon 7 strain MCCM 02309 is a member of the genus Pasteurella sensu stricto. $H$. parasuis MCCM $02736^{\mathbf{T}}$ had a polyamine pattern that was similar to the polyamine pattern obtained for $P$. pneumotropica type Jawetz strain MCCM $00234^{\mathrm{T}}$, which is a member of cluster 5 (see below). A close relationship between these two organisms is not supported by other data.

Cluster 4. Cluster 4 is subdivided into two subclusters. Most of the species belonging to Actinobacillus sensu stricto as defined by DNA relatedness data $(20,25)$ are members of subcluster 4A. Members of the genus Actinobacillus, including A. lignieresii MCCM $00190^{\mathrm{T}}$, Actinobacillus equuli MCCM $00142^{\mathrm{T}}$, A. capsulatus MCCM $02462^{\mathrm{T}}$, A. suis MCCM $02460^{\mathrm{T}}$, Actinobacillus ureae MCCM $00184^{\mathrm{T}}$, Actinobacillus hominis MCCM 00187 ${ }^{\mathrm{T}}$ and MCCM 00560, A. pleuropneumoniae MCCM $00339^{\mathrm{T}}$, and Actinobacillus sp. strain MCCM 00398 (Bisgaard taxon 9), had a polyamine pattern in which 1,3diaminopropane was the predominant polyamine. Other polyamines were not present or were found in minor amounts. This pattern was also detected in other strains of $A$. pleuropneumoniae belonging to biovars 1 and 2 , in $A$. lignieresii 9/91, and in an isolate of $A$. suis (Table 1). The other strain of $A$. ureae analyzed in this study, MCCM 02466, exhibited 100\% DNA reassociation with the type strain $(18,23)$; it contained 1,3diaminopropane as its major polyamine, but high concentrations of spermidine were also detected. This observation indicates that some variability in polyamine patterns occurs in $A$. ureae. A pattern characteristic of the genus Actinobacillus was detected in avian hemolytic Actinobacillus-like strain MCCM 01376 (Bisgaard taxon 26), which is considered an authentic member of the genus Actinobacillus (25), and in Bisgaard taxon 26 strain MCCM 01095. Haemophilus parahaemolyticus MCCM $00504^{\mathrm{T}}$, which clusters with the Actinobacillus group in subcluster $4 \mathrm{~A}$, was found to possess this pattern as well.

Similar polyamine patterns were obtained for the three representatives of subcluster $4 \mathrm{~B}, H$. ducreyi $\mathrm{MCCM} 00729^{\mathrm{T}}$ $P$. bettyae MCCM $00450^{\mathrm{T}}$, and $P$. haemolytica MCCM $00395^{\mathrm{T}}$ (Tables 2 and 3 ). However, in addition to the major compound 1,3-diaminopropane, significant amounts of spermidine and spermidine were detected in $H$. ducreyi MCCM $00729^{\mathrm{T}}$. These patterns allowed discrimination of $H$. ducreyi MCCM $00729^{\mathrm{T}}$ from the genus Haemophilus sensu stricto and discrimination of the two Pasteurella species from the genus Pasteurella sensu stricto.

$P$. haemolytica $\mathrm{A} 2683$ had a polyamine pattern similar to that of the type strain. $P$. haemolytica-like strain MCCM 00388 (Frederiksen's third taxon) and $P$. haemolytica-like strain MCCM 00594 (Frederiksen's fourth taxon) contained 1,3-diaminopropane as the major polyamine, but the spermidine and spermine contents of these organisms were significantly higher than the spermidine and spermine contents of $P$. haemolytica MCCM $00395^{\mathrm{T}}$. This resemblance in polyamine patterns indicates that there is some degree of relatedness between strains MCCM 00388 and MCCM 00594, although it has been demonstrated that these organisms belong to different species (17). A close relationship to members of other clusters, which might be indicated by the similarity in the polyamine patterns, is not supported by other data.

Cluster 5. $P$. pneumotropica type Jawetz groups with Actinobacillus muris in cluster 5 , and $P$. pneumotropica complex strain MCCM 01911 represents a distinct species that is closely related to $P$. pneumotropica MCCM $00234^{\mathrm{T}}$ (type Jawetz) (29). The phylogenetic position of $P$. pneumotropica type Heyl, which is also considered a distinct species (29), is still unclear. $P$. pneumotropica MCCM $00234^{\mathrm{T}}$, MCCM 01911, and MCCM 00237 could be distinguished from each other on the basis of differences in their polyamine patterns, whereas $P$. pneumotropica type Heyl strain MCCM 00253 had the same characteristic polyamine pattern as strain MCCM 00237 (Table 3). These observations indicate that the strains of $P$. pneumotropica type Jawetz and $P$. pneumotropica type Heyl may be distinguished by differences in their polyamine patterns. However, the polyamine patterns of the four strains studied are clearly different from the polyamine patterns which are representative of the genus Pasteurella sensu stricto, demonstrating that these organisms should be classified separately from the genus Pasteurella sensu stricto.

Cluster 6. $H$. parainfluenzae, which is considered a member of the genus Haemophilus sensu stricto (20), is the only species in cluster 6. This cluster is phylogenetically distant from $H$. influenzae, the type species of the genus, and the distance was supported by the distinct polyamine pattern (Table 2) obtained for $H$. parainfluenzae MCCM $00486^{\mathrm{T}}$. The phylogenetic position and the results of the polyamine analysis indicate that the taxonomic position of $H$. parainfluenzae MCCM $00486^{\mathrm{T}}$ should be reassessed.

Cluster 7. Pasteurella testudinis and Bisgaard taxon 14 strains are grouped in cluster 7. P. testudinis MCCM $00484^{\mathrm{T}}$ and MCCM 00400 had polyamine patterns in which spermidine was the predominant compound, and there were also relatively high concentrations of putrescine. The polyamine patterns clearly distinguished $P$. testudinis MCCM $00484^{\mathrm{T}}$ from the genus Pasteurella sensu stricto (Table 3). The similar polyamine pattern obtained for the two Bisgaard taxon 14 strains, MCCM 02121 and MCCM 02122 (Table 4), which are considered members of one species (2), is consistent with the close genetic 
relationship between these organisms and $P$. testudinis $\mathrm{MCCM}$ $00484^{\mathrm{T}}$. Osawa et al. (24) reported a similar polyamine pattern for Lonepinella koalarum, but in addition, they detected significant amounts of an unknown compound which we were not able to find in cluster 7 strains. The suggested genuslike structure consisting of $P$. testudinis $\mathrm{MCCM} 00484^{\mathrm{T}}$ and $P$. haemolytica MCCM 00395 ${ }^{\mathrm{T}}$ and MCCM 00388 (Frederiksen's third taxon) (17) is not supported by the differences in polyamine patterns (Tables 2 and 3 ) and the results of phylogenetic clustering based on $16 \mathrm{~S}$ rRNA sequence analyses (7).

Phylogenetically unassigned strains. So far, Haemophilus intermedius is considered a species that belongs to the genus Haemophilus sensu stricto (20). This species has not been included in $16 \mathrm{~S}$ rRNA sequence analyses. $H$. intermedius subsp. gazogenes MCCM 02082 was found to have a polyamine pattern similar to that obtained for $H$. parainfluenzae MCCM $00486^{\mathrm{T}}$ (cluster 6). This observation indicates that $H$. intermedius subsp. gazogenes MCCM 02082 and $H$. parainfluenzae MCCM $00486^{\mathrm{T}}$ may be closely related and does not support a close phylogenetic relationship to the genus Haemophilus sensu stricto. The distant relationship of $H$. intermedius to $H$. influenzae is also indicated by significant differences in quinone composition (9). Thus, reclassification of $H$. intermedius should be considered.

"Haemophilus felis" (14) and "Pasteurella leonis" have not been included in 16S rRNA studies. However, no relationship between " $\mathrm{H}$. felis" and some representatives of the genus Haemophilus was demonstrated by the results of DNA reassociation studies (14). The presence of significant amounts of symnorspermidine in the polyamine patterns (Tables 2 and 3) indicates that " $H$. felis" MCCM $02738^{\mathrm{T}}$ and MCCM 02064 and "P. leonis" might be members of subcluster $3 \mathrm{~B}$, the $P$. multocida group.

The results of DNA-rRNA studies have demonstrated that "Histophilus ovis" is a member of the family Pasteurellaceae (6). Similar polyamine patterns which varied only in the amounts of the minor compounds (Table 4) were obtained for "Histophilus ovis" MCCM 00330 (formerly "Haemophilus agni") and "Histophilus ovis" MCCM 02133 (formerly "Haemophilus somnus"), which can be considered strains of the same species (26). These patterns were similar to those obtained for subcluster $3 \mathrm{~A}$ and 3C strains. A high level of phylogenetic relatedness of "Histophilus ovis" to one of these two subclusters was not supported by the results of DNA-rRNA studies which included representatives of subcluster 3A (Pasteurella species A) and subcluster 3C (H. paracuniculus) (6). Also, the results of DNA-DNA hybridization studies which included "Histophilus ovis," $P$. avium, $P$. volantium, and Pasteurella species A did not reveal a relationship at the species level (26). The avian Haemophiluslike organism strain MCCM 01034 had a polyamine pattern (Table 4) which was most similar to the polyamine patterns obtained for subcluster $3 \mathrm{~A}$ and $3 \mathrm{C}$ strains and "Histophilus ovis" but the relationship of this organism to previously established taxa remains to be investigated.

The results of DNA reassociation studies have demonstrated that Actinobacillus rossii $\mathrm{MCCM} 02461^{\mathrm{T}}$ cannot be considered a member of the genus Actinobacillus (20). However, its polyamine pattern (Table 1) did not distinguish this species from Actinobacillus species. It can be assumed that this organism is not closely related to the genus Haemophilus sensu stricto because of differences in quinone composition (21).

The polyamine patterns of Pasteurella granulomatis MCCM $00576^{\mathrm{T}}$, Pasteurella caballi MCCM $00841^{\mathrm{T}}$, Pasteurella-like strain MCCM 00658, Pasteurella-like strain MCCM 00466 (hamster group 1), and Pasteurella-like strain MCCM 02120 (hamster group 2) clearly distinguished these organisms from the genus Pasteurella sensu stricto (Table 3), indicating that these strains should not be included in the genus Pasteurella sensu stricto. Relatedness to the genus Actinobacillus or Haemophilus or to other groups in the family characterized by the presence of 1,3-diaminopropane as the single polyamine in the polyamine pattern should be examined. Pasteurella-like strain MCCM 01664 had a polyamine pattern which contained putrescine as the major compound and high levels of 1,3-diaminopropane. This pattern was different from the patterns of species belonging to the genus Pasteurella sensu stricto, and it can be assumed that Pasteurella-like strain MCCM 01664 occupies a separate taxonomic position within the Pasteurellaceae.

Because the major compound 1,3-diaminopropane was detected in Bisgaard taxon 6 strains MCCM 02144 and MCCM 02143, Bisgaard taxon 20 strain MCCM 00674, and SP group strains MCCM 00489 and MCCM 02119 (Table 4), the possible relationship of these organisms to the genera Actinobacillus and Haemophilus should be investigated. Steward and Letscher strain MCCM 02118 and Bisgaard taxon 18 strain MCCM 02527 were characterized by a polyamine pattern (Table 4) which was similar to that obtained for members of subclusters $3 \mathrm{~A}$ and $3 \mathrm{C}$, but there are no other data which support a close relationship to one of these subclusters.

The polyamine pattern obtained for avian Haemophilus-like strain MCCM 02795 clearly distinguished this strain from the genus Haemophilus sensu stricto (Table 2). This polyamine pattern was most similar to the pattern obtained for $P$. aerogenes MCCM $00402^{\mathrm{T}}$, Bisgaard taxon 2 strain MCCM 00886 , and the two Bisgaard taxon 14 strains (see above). A possible relationship to any of these strains awaits investigation.

In conclusion, from the results obtained in this study it is obvious that the variability in polyamine patterns in the family Pasteurellaceae is much greater than the variability expected from studies of other gram-negative bacteria, in which polyamine patterns appear to be conserved and characteristic for higher taxa $(1,2,12,13)$. In the family Pasteurellaceae, at least seven different polyamine patterns were detected. Each of these patterns might be characteristic for a reclassified genus within the family Pasteurellaceae or for certain species of a genus. The variability does not seem to depend on the host from which a strain was isolated since members of the $P$. multocida group (subcluster $3 \mathrm{~B}$ ) characterized by similar polyamine patterns have been isolated from different mammalian species, including porcine, bovine, dog, and cat species, as well as humans (19). A similar observation was made for members of subcluster 3A. This subcluster consists of strains with a common polyamine pattern which have been isolated from several bird species $(19,22)$. Other isolates from mammals $(P$. bettyae and $P$. caballi) (16a) and birds ( $P$. anatis and $P$. langaa) $(19)$ had distinguishing polyamine patterns. These results demonstrate that the possible adaptation of a species belonging to the Pasteurellaceae to a host does not necessarily result in one particular polyamine pattern.

The importance of some of the polyamine patterns obtained for members of the family Pasteurellaceae has not been evaluated yet, since the phylogenetic relationships are not yet clear. Often, the phylogenetic branching of the strains within the family is rather deep, and different phylogenetic neighbors are found in different trees $(7,10,24)$. Closer phylogenetic relatives have to be found and analyzed to determine their polyamine patterns in order to evaluate the reliability of such patterns for use in the classification of these groups.

Some variability in the polyamine patterns of strains belonging to the same species appears to be possible, as shown for "Histophilus ovis" and $A$. ureae. This observation demonstrates that concentrations of certain polyamines that are less than 10 
TABLE 5. Phylogenetic clusters within the family Pasteurellaceae and the corresponding polyamine patterns

\begin{tabular}{|c|c|c|c|c|c|}
\hline \multirow[b]{2}{*}{ Organism $^{a}$} & \multirow{2}{*}{$\begin{array}{c}\text { Cluster of } \\
\text { Dewhirst et al. }\end{array}$} & \multirow{2}{*}{$\begin{array}{l}\text { Subcluster of } \\
\text { Dewhirst et al. }\end{array}$} & \multicolumn{3}{|c|}{ Polyamine patterns ${ }^{c}$} \\
\hline & & & $\begin{array}{c}\text { Major } \\
\text { compound(s) }\end{array}$ & $\begin{array}{l}\text { Characteristic minor } \\
\text { compound(s) }^{d}\end{array}$ & $\begin{array}{l}\text { Diagnostic } \\
\text { compound }\end{array}$ \\
\hline A. actinomycetemcomitans & 1 & $1 \mathrm{~A}$ & DAP & & \\
\hline H. aphrophilus & 1 & $1 \mathrm{~B}$ & DAP & & \\
\hline H. segnis & 1 & $1 \mathrm{~B}$ & DAP & & \\
\hline H. influenzae & 1 & $1 \mathrm{C}$ & DAP & & \\
\hline H. aegyptius & 1 & $1 \mathrm{C}$ & DAP & & \\
\hline A. seminis & 2 & $2 \mathrm{~A}$ & DAP, PUT & SPM, CAD & \\
\hline P. mairii & 2 & $2 \mathrm{~A}$ & DAP, PUT & CAD & \\
\hline P. aerogenes & 2 & $2 \mathrm{~A}$ & SPD & PUT & \\
\hline H. paragallinarum & 3 & $3 \mathrm{~A}$ & DAP & SPD & \\
\hline P. avium & 3 & $3 \mathrm{~A}$ & DAP & SPD & \\
\hline$P$. volantium & 3 & $3 \mathrm{~A}$ & DAP & SPD & \\
\hline P. gallinarum & 3 & $3 \mathrm{~A}$ & DAP & SPD & \\
\hline P. multocida & 3 & 3B & PUT & SPD & NSPD \\
\hline P. canis & 3 & $3 \mathrm{~B}$ & SPD, PUT & & NSPD \\
\hline P. dagmatis & 3 & $3 B$ & PUT, DAP & SPD & NSPD \\
\hline P. stomatis & 3 & 3B & PUT, SPD & & NSPD \\
\hline Pasteurella species B & 3 & $3 \mathrm{~B}$ & SPD & PUT & NSPD \\
\hline Bisgaard taxon 7 & 3 & $3 \mathrm{C}$ & DAP & & \\
\hline H. paracuniculus & 3 & $3 \mathrm{C}$ & DAP & SPD & \\
\hline A. capsulatus & 3 & $3 C$ & DAP & & \\
\hline P. trehalosi & 3 & $3 \mathrm{C}$ & DAP & & \\
\hline H. parasuis & 3 & $3 \mathrm{C}$ & DAP, PUT & & \\
\hline$P$. langaa & 3 & $3 \mathrm{D}$ & DAP & & \\
\hline Bisgaard taxon 2 & 3 & $3 \mathrm{D}$ & SPD & PUT & \\
\hline P. anatis & 3 & $3 \mathrm{D}$ & DAP & PUT, SPD & \\
\hline "A. salpingitidis" & 3 & $3 \mathrm{D}$ & DAP & SPD & \\
\hline A. equuli & 4 & $4 \mathrm{~A}$ & DAP & & \\
\hline A. suis & 4 & $4 \mathrm{~A}$ & DAP & & \\
\hline A. hominis & 4 & $4 \mathrm{~A}$ & DAP & & \\
\hline A. ureae & 4 & $4 \mathrm{~A}$ & DAP & & \\
\hline A. lignieresii & 4 & $4 \mathrm{~A}$ & DAP & & \\
\hline A. pleuropneumoniae & 4 & $4 \mathrm{~A}$ & DAP & & \\
\hline H. parahaemolyticus & 4 & $4 \mathrm{~A}$ & DAP & & \\
\hline Bisgaard taxon 9 & 4 & $4 B$ & DAP & SPD & \\
\hline H. ducreyi & 4 & 4B & DAP & SPD & \\
\hline$P$, bettii & 4 & 4B & DAP & & \\
\hline P. haemolytica & 4 & $4 B$ & DAP & & \\
\hline P. pneumotropica & 5 & & DAP, PUT & & \\
\hline$P$. parainfluenzae & 6 & & $\mathrm{DAP}, \mathrm{CAD}$ & PUT & \\
\hline P. testudinis & 7 & & SPD, PUT & & \\
\hline Bisgaard taxon 14 & 7 & & SPD, PUT & & \\
\hline
\end{tabular}

a Organisms which have been phylogenetically investigated (7) and analyzed to determine their polyamine patterns are listed.

${ }^{b}$ See reference 7.

${ }^{c}$ For abbreviations see Table 1 , footnote $a$. Compounds are listed in order of decreasing concentration.

${ }^{d}$ Polyamines which account for more than $25 \%$ of the total polyamines are considered major compounds, whereas polyamines which account for 9 to $25 \%$ of the total polyamines are considered characteristic minor compounds.

$\mu \mathrm{mol} / \mathrm{g}$ (dry weight) of cells are sometimes difficult to interpret. An increase in the amount of a polyamine, such as the amount of spermidine in "Histophilus ovis" MCCM 00330 and A. ureae MCCM 02466, can lead to a change in the polyamine pattern to a pattern that is characteristic for other clusters. Thus, the results of polyamine analyses should be integrated in a polyphasic approach for classification of members of the family Pasteurellaceae. Nevertheless, some findings important for classification of members of the Pasteurellaceae are ob- tained solely from analyses of the polyamine patterns (Table 5).

The type species of the genera Actinobacillus and Haemophilus and their phylogenetic neighbors have a polyamine pattern in which 1,3-diaminopropane is the predominant compound. This pattern was found in many members of the family belonging to different clusters. Thus, the value of this characteristic for classification within the family is limited. However, species that are considered members of the genus Actinobacillus or 
Haemophilus should contain 1,3-diaminopropane as the predominant compound. Species and subspecies of these genera which are characterized by other patterns should be investigated to determine whether they can be considered members of the genera to which they are assigned; examples of such taxa are $A$. seminis, $H$. ducreyi, $H$. intermedius subsp. gazogenes, $H$. paracuniculus, $H$. paragallinarum, $H$. parainfluenzae, and $H$. parasuis, which have distinguishing polyamine patterns.

The differences in the polyamine patterns of members of the genus Pasteurella sensu stricto support the suggestion of Dewhirst et al. (7) that taxa included in the genus Pasteurella sensu stricto as defined by Mutters et al. (20) represent more than one genus. The following four genera are indicated based solely on different polyamine patterns: genus 1 , which is characterized by high levels of putrescine and spermidine and the presence of sym-norspermidine (subcluster 3B, possibly including "P. leonis" and "H. felis"); genus 2 , which is characterized by high levels of 1,3-diaminopropane and spermidine (subcluster $3 \mathrm{~A}$ ); genus 3 , which is characterized by the predominance of 1,3-diaminopropane ( $P$. langaa); and genus 4 , which is characterized by high levels of 1,3-diaminopropane, putrescine, and spermidine ( $P$. anatis).

Another genuslike structure may be created for members of cluster 7 (P. testudinis and strains belonging to Bisgaard taxon $14)$, which are characterized by a polyamine pattern in which the predominant compound is spermidine and high levels of putrescine are present.

The results of our investigations of polyamine patterns agree better with the groups based on the results of 16S rRNA sequence analyses (7) than with the taxa based on DNA reassociation values. On the other hand, the polyamine patterns of the representatives of some subclusters, such as subclusters $2 \mathrm{~A}$, $3 \mathrm{C}, 3 \mathrm{D}$, and 4B, are not homogeneous. This may be an indication that within these groups the polyamine approach is not suitable for classification which is in agreement with the phylogenetic clusters or that the phylogenetic branching needs to be reassessed. However, more genetic and chemotaxonomic data have to be obtained for reliable reclassification of the genera within the family and for descriptions of the new taxa which were revealed by the different polyamine patterns obtained for members of the family Pasteurellaceae.

\section{ACKNOWLEDGMENTS}

H.-J.B. is indebted to the Austrian Science Foudation for Lise Meitner fellowship M00159-MOB.

We gratefully acknowledge the excellent technical assistance of Maria Stadler. We thank F. Eko for critically reading the manuscript, W. Mannheim for supplying a large number of strains, and R. Mutters for supplying important information concerning certain strains.

\section{REFERENCES}

1. Auling, G., H.-J. Busse, F. Pilz, L. Webb, H. Kneifel, and D. Claus. 1991 Rapid differentiation by polyamine analysis of Xanthomonas strains from phytopathogenic pseudomonads and other members of the class Proteobacteria interacting with plants. Int. J. Syst. Bacteriol. 41:223-228.

2. Bisgaard, M., and R. Mutters. 1986. A new facultatively anaerobic Gramnegative fermentative rod obtained from different pathological lesions in poultry and tentatively designated taxon 14. Avian Pathol. 15:117-127.

3. Brumpt, E. 1910. Précis de parasitologie, 1st ed. Masson et Co., Paris, France.

4. Busse, J., and G. Auling. 1988. Polyamine pattern as a chemotaxonomic marker within the Proteobacteria. Syst. Appl. Microbiol. 11:1-8.

5. Chuba, P. J., R. Bock, G. Graf, T. Adam, and U. Göbel. 1988. Comparison of 16S rRNA sequences from the family Pasteurellaceae: phylogenetic relatedness by cluster analysis. J. Gen. Microbiol. 134:1923-1930.

6. De Ley, J., W. Mannheim, R. Mutters, K. Piechulla, R. Tytgat, P. Segers, M. Bisgaard, W. Frederiksen, K.-H. Hinz, and M. Vanhoucke. 1990. Inter- and intrafamilial similarities of rRNA cistrons of the Pasteurellaceae. Int. J. Syst. Bacteriol, 40:126-137.

7. Dewhirst, F. E., B. J. Paster, I. Olsen, and G. J. Fraser. 1993. Phylogeny of the Pasteurellaceae as determined by comparison of $16 \mathrm{~S}$ ribosomal ribonucleic acid sequences. Zentralbl. Bakteriol. Parasitenkd. Infektionskr. Hyg. Abt. 1 Orig. 279:35-44.

8. Engelhard, E., R. M. Kroppenstedt, R. Mutters, and W. Mannheim. 1991. Carbohydrate patterns, cellular lipoquinones, fatty acids and phospholipids of the genus Pasteurella sensu stricto. Med. Microbiol. Immunol. 180:79-92.

9. Engelhard, E., R. M. Kroppenstedt, R. Mutters, and W. Mannheim. 1992. Cytochemische Charakterisierung der humanen Haemophilus-Spezies sowie von (Actinobacillus) actinomycetemcomitans, p. 273-288. In L. Flores-deJacoby and W. Mannheim (ed.), 2nd Workshop Immunologie und Mikrobiologie der parodontalen Erkrankungen. Quintessenz-Verlag, Berlin, Germany.

10. Foster, G., H. M. Ross, H. Malnick, A. Willems, P. Garcia, R. J. Reid, and M. D. Collins. 1996. Actinobacillus delphinicola sp. nov., a new member of the family Pasteurellaceae Pohl (1979) 1981 isolated from sea mammals. Int J. Syst. Bacteriol. 46:648-652.

11. Frederiksen, W. 1973. Pasteurella taxonomy and nomenclature, p. 170-176 In $\mathrm{S}$. Winblad (ed.), Contributions to microbiology and immunology, vol. 2. Yersinia, Pasteurella and Francisella. Karger, Basel, Switzerland.

12. Hamana, K., and S. Matsuzaki. 1990 . Occurrence of homospermidine as a major polyamine in the authentic genus Flavobacterium. Can. J. Microbiol 36:228-231.

13. Hamana, K., and S. Matsuzaki. 1991. Polyamine distribution in the Flavobacterium-Cytophaga-Sphingobacterium complex. Can. J. Microbiol. 37:885888.

14. Inzana, T. J., J. L. Johnson, L. Shell, K. Møller, and M. Kilian. 1992 Isolation and characterization of a newly identified Haemophilus species from cats: "Haemophilus felis." J. Clin. Microbiol. 30:2108-2112.

15. Kroppenstedt, R. M., and W. Mannheim. 1989. Lipoquinones in members of the family Pasteurellaceae. Int. J. Syst. Bacteriol. 39:304-308.

16. Mannheim, W. 1981. Taxonomic implications of DNA relatedness and quinone patterns in Actinobacillus, Haemophilus, and Pasteurella, p. 265-280. In M. Kilian, W. Frederiksen, and E. L. Biberstein (ed.), Haemophilus, Pasteurella, and Actinobacillus. Academic Press, Inc., London, England.

16a.Mannheim, W. Personal communication.

17. Mutters, R., M. Bisgaard, and S. Pohl. 1986. Taxonomic relationship of selected biogroups of Pasteurella haemolytica as revealed by DNA:DNA hybridizations. Acta Pathol. Microbiol. Immunol. Scand. Sect. B 94:195-202.

18. Mutters, R., W. Frederiksen, and W. Mannheim. 1984. Lack of evidence for the occurrence of Pasteurella ureae in rodents. Vet. Microbiol. 9:83-93.

19. Mutters, R., P. Ihm, S. Pohl, W. Frederiksen, and W. Mannheim. 1985. Reclassification of the genus Pasteurella Trevisan 1887 on the basis of deoxyribonucleic acid homology, with proposal for the new species Pasteurella dagmatis, Pasteurella canis, Pasteurella stomatis, Pasteurella anatis, and Pasteurella langaa. Int. J. Syst. Bacteriol. 35:309-322.

20. Mutters, R., W. Mannheim, and M. Bisgaard. 1989. Taxonomy of the group, p. 3-34. In C. Adlam and J. M. Rutter (ed.), Pasteurella and pasteurellosis. Academic Press, Inc. (London), Ltd., London, England.

21. Mutters, R., M. Mouhahid, E. Engelhard, and W. Mannheim. 1993. Characterization of the family Pasteurellaceae on the basis of cellular lipids and carbohydrates. Zentralbl. Bakteriol. Parasitenkd. Infektionskr. Hyg. Abt. 1 Orig. 279: 104-113.

22. Mutters, R., K. Piechulla, K.-H. Hinz, and W. Mannheim. 1985. Pasteurella avium (Hinz and Kunjara) comb. nov. and Pasteurella volantium sp. nov. Int J. Syst. Bacteriol. 35:5-9.

23. Mutters, R., S. Pohl, and W. Mannheim. 1986. Transfer of Pasteurella ureae Jones 1962 to the genus Actinobacillus Brumpt 1910: Actinobacillus ureae comb. nov. Int. J. Syst. Bacteriol. 36:343-344.

24. Osawa, R., F. Rainey, T. Fujisawa, E. Lang, H. J. Busse, T. P. Walsh, and E Stackebrandt. 1995. Lonepinella koalarum gen. nov., sp. nov., a new tanninprotein complex degrading bacterium. Syst. Appl. Microbiol. 18:368-373.

25. Piechulla, K., M. Bisgaard, H. Gerlach, and W. Mannheim. 1985. Taxonomy of some recently described avian Pasteurella/Actinobacillus-like organisms as indicated by deoxyribonucleic acid relatedness. Avian Pathol 14:281-311.

26. Piechulla, K., R. Mutters, S. Burbach, R. Klussmeier, S. Pohl, and W. Mannheim. 1986. Deoxyribonucleic acid relationships of "Histophilus ovis/ Haemophilus somnus," Haemophilus haemoglobinophilus, and "Actinobacillus seminis." Int. J. Syst. Bacteriol. 36:1-7.

27. Pohl, S., H. U. Bertschinger, W. Frederiksen, and W. Mannheim. 1983. Transfer of Haemophilus pleuropneumoniae and the Pasteurella haemolyticalike organism causing porcine necrotic pleuropneumonia to the genus Actinobacillus (Actinobacillus pleuropneumoniae comb. nov.) on the basis of phenotypic and deoxyribonucleic acid relatedness. Int. J. Syst. Bacteriol. 33:510-514.

28. Potts, T. V., J. J. Zambon, and R. J. Genco. 1985. Reassignment of Actinobacillus actinomycetemcomitans to the genus Haemophilus as Haemophilus actinomycetemcomitans comb. nov. Int. J. Syst. Bacteriol. 35:337-341.

29. Ryll, M. R. Mutters, and W. Mannheim. 1991. Untersuchungen zur genetischen Klassifikation des Pasteurella-pneumotropica-Komplexes. Berl. Muench. Tieraerztl. Wochenschr. 104:243-245.

30. Sneath, P. H. A., and R. Johnson. 1973. Numerical taxonomy of Haemophilus and related bacteria. Int. J. Syst. Bacteriol. 23:405-418. 
31. Trevisan, V. 1887. Sul micrococco della rabbia e sulla possibilità di reconoscere durante il periodo d'incubazione, dall'esame del sangue della persona moricata, se ha contratta 1 ínfezione rabica. Rend. Ist. Lombardo Ser. 2 20:88-105.

32. Winslow, C.-E. A., J. Broadhurst, R. E. Buchanan, C. Krumwiede, Jr., L. A. Rogers, and G. H. Smith. 1917. The families and genera of the bacteria.
Preliminary report of the Committee of the Society of American Bacteriologists on Characterization and Classification of Bacterial Types. J. Bacteriol. 2:505-566

33. Woese, C. R., W. G. Weisburg, C. M. Hahn, B. J. Paster, L. B. Zablen, B. J. Lewis, T. J. Macke, W. Ludwig, and E. Stackebrandt. 1985. The phylogeny of purple bacteria: the gamma subdivision. Syst. Appl. Microbiol. 6:25-33. 